\title{
Continuous embedding between P-de Branges spaces
}

https://doi.org/10.1515/conop-2020-0118

Received March 4, 2021; accepted July 21, 2021

Abstract: In this paper we study the continuity of the embedding operator $\iota: \mathcal{H}^{p}(E) \hookrightarrow \mathcal{H}^{q}(E)$ when $0<p<$ $q \leqslant \infty$. The necessary and sufficient condition has already been described in [10] if $p>1$. In this work, we address the problem when $p=1$, using a new approach, but asking some additional hypothesis about the Hermite-Biehler function $E$. We give also a different proof for the case $p>1$.

Keywords: de Branges spaces, continuous embedding operator

MSC: $30 \mathrm{H} 99$

\section{Introduction}

The continuity of the embedding operator between different $p$-Bernstein spaces is a well known result, [1] and [14]. In these notes, we study the same problem for the de Branges spaces: we ask which conditions the Hermite-Biehler function $E$ must satisfy so that the embedding operator

$$
\iota: \mathcal{H}^{p}(E) \hookrightarrow \mathcal{H}^{q}(E) \quad \text { when } 0<p<q \leqslant \infty
$$

is continuous.

This problem has already been investigated and solved in [8], [9], [10] by Professor K. Dyakonov, for $p>1$. Dyakonov proves that the boundedness of the derivative of the phase function of the meromorphic inner function $\Theta$ is a necessary and sufficient condition for the continuity of the embedding operator in $*$-invariant subspaces $\mathcal{K}^{p}(\Theta)$ of Hardy space. This condition can be adapted also to de Branges spaces.

Instead of focusing on the case $p>1$, we fix our attention to the case $p=1$, which was already been studied in [9] only for some kind of meromorphic inner functions. We prove that

$$
\left\|\phi^{\prime}\right\|_{L^{\infty}}<\infty
$$

if and only if the embedding operator

$$
\iota: \mathcal{H}^{1}(E) \hookrightarrow \mathcal{H}^{q}(E)
$$

is continuous. However, to prove the theorem, we add further conditions to $E$, (2.5) and (2.6).

For the case $p>1$, we present a proof longer than the theorem of Dyakonov. However, since it is similar and it uses the same approach to the case $p=1$, we write it down. Furthermore, the whole reasoning can also be generalized to the case $1 / 2<p<1$.

These notes are divided in four short sections. The next section is introductory: we recall the main properties of the $p$-de Branges spaces. We skip almost all the details for which we refer to the classic texts on the subjects, such as [5], [6], [11], [14], [16] or [17]. At the end of this section, we state the two main results: Theorem 2.4 and Theorem 2.5. In the third section we prove Theorem 2.4 and we will highlight where our proof is different from that of [10]. In the fourth section, we study the necessary condition for $p=1$ and we prove Theorem 2.5.

${ }^{\star}$ Corresponding Author: Carlo Bellavita : Department of Mathematics, Università degli Studi di Milano, Milano, Italy, E-mail: carlo.bellavita@unimi.it 


\section{$2 \boldsymbol{p}$-de Branges spaces}

$p$-de Branges spaces are widely described, for instance, in [3], [4], [12], [13]. Here we recall their first properties. DEFinition 2.1 The entire function $E$ is a Hermite-Biehler function if

$$
|E(\bar{z})|<|E(z)| \quad \text { for every } z \in \mathbb{C}^{+} .
$$

Definition 2.2 Let $0<p \leqslant \infty$. Given the Hermite-Biehler function $E$, the $p$-de Branges space $\mathcal{H}^{p}(E)$ is defined as:

$$
\mathcal{H}^{p}(E):=\left\{f \text { entire such that } f / E \in H^{p}\left(\mathbb{C}^{+}\right), f^{\#} / E \in H^{p}\left(\mathbb{C}^{+}\right)\right\},
$$

where $f^{\#}(z):=\overline{f(\bar{z})}$ and $H^{p}\left(\mathbb{C}^{+}\right)$is the $p$-Hardy space of $\mathbb{C}^{+}$.

If $p \geqslant 1, \mathcal{H}^{p}(E)$ is a Banach space with the norm inherited from $H^{p}\left(\mathbb{C}^{+}\right)$

$$
\|f\|_{\mathcal{H}^{p}}:=\|f / E\|_{H^{p}}=\left(\int_{\mathbb{R}}\left|\frac{f(x)}{E(x)}\right|^{p} d x\right)^{1 / p} .
$$

If $0<p<1, \mathcal{H}^{p}(E)$ is just a complete metric space and the distance is given by

$$
d(f, g)=\|f-g\|_{H^{p}}=\left(\int_{\mathbb{R}}\left|\frac{f(x)-g(x)}{E(x)}\right|^{p} d x\right) .
$$

Every Hermite-Biehler function $E$ can be associated to a meromorphic inner functions $\Theta$, as shown in [15]; Indeed, if $E$ is a Hermite-Biehler function, then

$$
\Theta:=E^{\#} / E
$$

is a meromorphic inner function of $\mathbb{C}^{+}$. Therefore, if $p>1$, the $p$-de Branges spaces are isometrically isomorphic to the $*-p$ invariant subspaces of $H^{p}\left(\mathbb{C}^{+}\right)$, as proved for example in [2] when $p=2$. The proof of Dyakonov in [10] is settled for $*-p$ invariant subspaces.

When dealing with de Branges spaces, the phase function is an important instrument.

DEFINITION 2.3 We denote by $\phi(x)$ the phase function associated to $E(z)$, that is, the continuous function such that $e^{i \phi(x)} E(x)=|E(x)|$ for all real $x$.

It can be shown that $\phi(z)$ has an analytic continuation to an open neighborhood of $\mathbb{R}$ and it is possible to compute explicitly the expression of its derivative. Indeed, let $\left\{z_{n}:=a_{n}-i b_{n}\right\}_{n \in \mathbb{Z}}$ be the zeros of the HermiteBiehler function, then

$$
\phi^{\prime}(t):=\sum_{n \in \mathbb{Z}} \frac{b_{n}}{\left(t-a_{n}\right)^{2}+b_{n}^{2}},
$$

where we know that the above series converges when $t$ is fixed due to the Blaschke condition [7]. If $p \geqslant 1$, the spaces $\mathcal{H}^{p}(E)$ are reproducing kernel Banach spaces. The kernel is defined as

$$
k_{z}(t):=\frac{i}{2 \pi} \frac{E(t) \overline{E(z)}-E^{\#}(t) E(\bar{z})}{\bar{z}-t} \quad z, t \in \mathbb{C} .
$$

If $p>1, k_{z}(t)$ belongs to $\mathcal{H}^{p}(E)$ for every $z \in \mathbb{C}$.

We are now ready for our main results which we prove in the next sections.

THEOREM 2.4. Let $1<p<q \leqslant \infty$ and let $E$ be a Hermite-Biehler function. The embedding operator $\iota$ : $\mathcal{H}^{p}(E) \hookrightarrow \mathcal{H}^{q}(E)$ is continuous if and only if $\left\|\phi^{\prime}\right\|_{\infty}<\infty$. 
THEOREM 2.5. Let $\left\{z_{n}=a_{n}-i b_{n}\right\}_{n \in \mathbb{Z}}$ be the zeros of the Hermite-Biehler function E. Let the derivative of the phase function $\phi^{\prime}(x)$ be uniformly bounded away from zero, that is

$$
\inf _{x \in \mathbb{R}} \phi^{\prime}(x) \geqslant \delta>0
$$

and let

$$
\inf _{n \in \mathbb{Z}}\left|b_{n}\right| \geqslant M>0 .
$$

$\iota: \mathcal{H}^{1}(E) \hookrightarrow \mathcal{H}^{q}(E)$ is continuous if and only if $\left\|\phi^{\prime}\right\|_{\infty}<\infty$.

\section{Proof of Theorem 2.4}

In this section we focus our attention on the necessary and sufficient condition for the continuity of the embedding operator when $p>1$. The proof that we present works directly at the level of the de Branges spaces. Let us start with sufficiency. We need this preliminary lemma.

LEMMA 3.1. Let $\phi^{\prime}$, the derivative of the Hermite-Biehler function $E$, be bounded on $\mathbb{R}$ and let $p \geqslant 1$. Then for every $f \in \mathcal{H}^{p}(E)$ and for every $x \in \mathbb{R}$

$$
\left|\frac{f(x)}{E(x)}\right| \leqslant C(E, p)\|f\|_{\mathcal{H}^{p}}\left(1+\frac{1}{\inf _{\xi \in \mathbb{R}, 0<\eta<\tau}|\Theta(\xi+i \eta)|^{p}}\right)^{1 / p},
$$

where $\Theta$ is the meromorphic inner function associated to the Hermite-Biehler function E. $\tau$ depends only on $E$ and is specified in the proof.

Proof. Thanks to Lemma 1 of [10], if $\phi^{\prime} \in L^{\infty}(\mathbb{R})$, then for some $\delta>0$,

$$
\inf \{|\Theta(z)|: 0<\Im z<\delta\}>0 \text { where } \Theta=E^{\#} / E .
$$

Due to the subharmonicity of $|f / E|^{p}$ in a strip which contains the real line, we know that

$$
\left|\frac{f(x)}{E(x)}\right| \leqslant\left(\frac{1}{\pi \tau^{2}} \int_{B_{\tau}(x)}\left|\frac{f(\xi)}{E(\xi)}\right|^{p} d \xi\right)^{1 / p} \quad \text { if } \tau<\delta .
$$

Thus, we obtain that

$$
\begin{aligned}
\left|\frac{f(x)}{E(x)}\right| & \leqslant\left(\frac{1}{\pi \tau^{2}} \int_{-\tau}^{\tau} \int_{\mathbb{R}}\left|\frac{f(x+\xi+i \eta)}{E(x+\xi+i \eta)}\right|^{p} d \xi d \eta\right)^{1 / p} \\
& =\left[\frac{1}{\pi \tau^{2}}\left(\int_{0}^{\tau} \int_{\mathbb{R}}\left|\frac{f(\xi+i \eta)}{E(\xi+i \eta)}\right|^{p} d \xi d \eta+\int_{-\tau}^{0} \int_{\mathbb{R}}\left|\frac{f(\xi+i \eta)}{E(\xi+i \eta)}\right|^{p} d \xi d \eta\right)\right]^{1 / p} \\
& \leqslant\left[\frac{1}{\pi \tau^{2}}\left(\tau\|f\|_{\mathcal{H}^{p}}^{p}+\int_{-\tau}^{0} \int_{\mathbb{R}}\left|\frac{f(\xi+i \eta)}{E(\xi-i \eta)}\right|^{p}\left|\frac{E(\xi-i \eta)}{E(\xi+i \eta)}\right|^{p} d \xi d \eta\right)\right]^{1 / p} \\
& \leqslant\left[\frac{1}{\pi \tau^{2}}\left(\tau\|f\|_{\mathcal{H}^{p}}^{p}+\sup _{\xi, \eta}\left|\frac{E(\xi-i \eta)}{E(\xi+i \eta)}\right|^{p} \int_{-\tau}^{0} \int_{\mathbb{R}}\left|\frac{f^{\#}(\xi-i \eta)}{E(\xi-i \eta)}\right|^{p} d \xi d \eta\right)\right]^{1 / p} \\
& \leqslant\left[\frac{1}{\pi \tau^{2}}\left(\tau\|f\|_{\mathcal{H}^{p}}^{p}+\left.\sup _{\xi \in \mathbb{R}, 0<-\eta<\tau} \frac{1}{\left|\frac{E^{\sharp}(\xi-i \eta)}{E(\xi-i \eta)}\right|^{p}} \tau|| f^{\#}\right|_{\mathcal{H}^{p}} ^{p}\right)\right]^{1 / p} \\
& =\left(\frac{1}{\pi \tau}\right)^{1 / p}\|\|_{\mathcal{H}^{p}}\left(1+\frac{1}{\inf _{\xi \in \mathbb{R}, 0<\eta<\tau}|\Theta(\xi+i \eta)|^{p}}\right)^{1 / p} .
\end{aligned}
$$


Proof Sufficiency 2.4. At this point, the proof is an easy consequence of (3.1). We recall that if $f \in H^{p}\left(\mathbb{C}^{+}\right) \cap$ $L^{q}(\mathbb{R})$, then $f \in H^{q}\left(\mathbb{C}^{+}\right)$. Consequently we have just to check the relation between the two norms. For every $f \in \mathcal{H}^{p}$, we note that

$$
\begin{aligned}
\left(\int_{\mathbb{R}}\left|\frac{f(x)}{E(x)}\right|^{q} d x\right)^{1 / q} & \leqslant\left(\int_{\mathbb{R}}\left|\frac{f(x)}{E(x)}\right|^{p} d x\right)^{1 / q}\left(\sup _{x \in \mathbb{R}}\left|\frac{f(x)}{E(x)}\right|^{q-p} d x\right)^{1 / q} \\
& \leqslant\|f\|_{\mathcal{H}^{p}}^{p / q} C(E, p, q)\|f\|_{\mathcal{H}^{p}}^{1-p / q} \leqslant C\|f\|_{\mathcal{H}^{p}},
\end{aligned}
$$

with the natural changes if $q=\infty$.

REMARK 3.2 We specify that the proof of the sufficiency condition is similar to that of [10]. The main difference is the use of Lemma 3.1, instead of some estimates on the reproducing kernel. We think that this lemma is more similar to the techniques used in Bernstein spaces.

Now, we move on to the proof of the necessary condition for the continuity of the embedding operator $\mathcal{H}^{p}(E) \hookrightarrow \mathcal{H}^{q}(E)$ when $1<p<q \leqslant \infty$. We need some preliminaries estimates.

Proposition 3.3. Let $\left\{z_{n}=a_{n}-i b_{n}\right\}_{n \in \mathbb{Z}}$ be the zeros of the Hermite-Biehler function E. If ı : $\mathcal{H}^{p}(E) \hookrightarrow$ $\mathcal{H}^{q}(E)$ is continuous, then there exists $M>0$ such that (2.6) holds.

Proof. Let us consider

$$
k_{\overline{z_{n}}}(t)=\frac{i}{2 \pi} \frac{E(t) E^{\#}\left(z_{n}\right)-E^{\#}(t) E\left(z_{n}\right)}{z_{n}-t}=\frac{i}{2 \pi} \frac{E(t) E^{\#}\left(z_{n}\right)}{z_{n}-t},
$$

since $E\left(z_{n}\right)=0$ for every $n \in \mathbb{Z}$. Since $\iota$ is continuous

$$
\begin{aligned}
\frac{\left|E^{\#}\left(z_{n}\right)\right|}{2 \pi}\left(\int_{\mathbb{R}} \frac{1}{\left|z_{n}-t\right|^{q}} d t\right)^{1 / q} & =\left\|k_{\overline{z_{n}}}\right\|_{\mathcal{H}^{q}} \\
& \leqslant C\left\|k_{\overline{z_{n}}}\right\|_{\mathcal{H}^{p}}=C \frac{\left|E^{\#}\left(z_{n}\right)\right|}{2 \pi}\left(\int_{\mathbb{R}} \frac{1}{\left|z_{n}-t\right|^{p}} d t\right)^{1 / p}
\end{aligned}
$$

and consequently

$$
b_{n}^{1 / q-1} \leqslant C^{\prime} b_{n}^{1 / p-1}
$$

that is

$$
b_{n}^{1 / q-1 / p} \leqslant C^{\prime}
$$

for every $n \in \mathbb{Z}$, which implies that the $\inf _{n \in \mathbb{Z}} b_{n}>0$.

In the following estimates we will repeatedly use condition (2.6) and the constant $M$ will be used for the lower bound of imaginary parts of the zeros of $E$.

LEMMA 3.4. Let $\iota: \mathcal{H}^{p}(E) \hookrightarrow \mathcal{H}^{q}(E)$ be continuous. It holds that

$$
\phi^{\prime}(s) \leqslant 4 \phi^{\prime}(t) \quad \text { for everys such that }|s-t| \leqslant M .
$$

Proof. We know that

$$
\begin{aligned}
\phi^{\prime}(s)=\sum_{n} \frac{b_{n}}{\left(a_{n}-s\right)^{2}+b_{n}^{2}} \leqslant \sum_{n} \frac{b_{n}}{\left(a_{n}-t\right)^{2}+b_{n}^{2}} \sup _{n} \frac{\left(a_{n}-t\right)^{2}+b_{n}^{2}}{\left(a_{n}-s\right)^{2}+b_{n}^{2}} \\
\quad \leqslant \sum_{n} \frac{b_{n}}{\left(a_{n}-t\right)^{2}+b_{n}^{2}} \sup _{n}\left(1+\frac{(s-t)^{2}+2\left|(s-t)\left(a_{n}-s\right)\right|}{\left(a_{n}-s\right)^{2}+b_{n}^{2}}\right) .
\end{aligned}
$$


If $\left|a_{n}-s\right|<b_{n}$

$$
\begin{aligned}
\sup _{n}\left(1+\frac{(s-t)^{2}+2\left|(s-t)\left(a_{n}-s\right)\right|}{\left(a_{n}-s\right)^{2}+b_{n}^{2}}\right) & \leqslant \sup _{n}\left(1+\frac{M^{2}+2 M b_{n}}{b_{n}^{2}}\right) \\
& \leqslant \sup _{n}\left(1+\frac{b_{n}^{2}+2 b_{n}^{2}}{b_{n}^{2}}\right)=4 .
\end{aligned}
$$

On the other hand, if $\left|a_{n}-s\right| \geqslant b_{n} \geqslant M$

$$
\begin{aligned}
& \sup _{n}\left(1+\frac{(s-t)^{2}+2\left|(s-t)\left(a_{n}-s\right)\right|}{\left(a_{n}-s\right)^{2}+b_{n}^{2}}\right) \\
& \quad \leqslant \sup _{n}\left(1+\frac{M^{2}+2 M\left|a_{n}-s\right|}{\left(a_{n}-s\right)^{2}}\right) \leqslant \sup _{n}\left(1+\frac{\left(a_{n}-s\right)^{2}+2\left(a_{n}-s\right)_{n}^{2}}{\left(a_{n}-s\right)^{2}}\right)=4 .
\end{aligned}
$$

Therefore in any cases, we have (3.3).

LEMMA 3.5. Let $\iota: \mathcal{H}^{p}(E) \hookrightarrow \mathcal{H}^{q}(E)$ be continuous. It holds that

$$
\phi^{\prime}(s) \geqslant \frac{1}{2} \phi^{\prime}(t) \quad \text { for everys such that }|s-t| \leqslant \frac{M}{4} .
$$

Proof. We know that

$$
\phi^{\prime}(s)=\sum_{n} \frac{b_{n}}{\left(a_{n}-s\right)^{2}+b_{n}^{2}} \geqslant \sum_{n} \frac{b_{n}}{\left(a_{n}-t\right)^{2}+b_{n}^{2}} \inf _{n} \frac{\left(a_{n}-t\right)^{2}+b_{n}^{2}}{\left(a_{n}-s\right)^{2}+b_{n}^{2}} .
$$

If $\left|a_{n}-s\right| \leqslant b_{n}$

$$
\inf _{n} \frac{\left(a_{n}-t\right)^{2}+b_{n}^{2}}{\left(a_{n}-s\right)^{2}+b_{n}^{2}} \geqslant \inf _{n} \frac{b_{n}^{2}}{2 b_{n}^{2}}=\frac{1}{2} .
$$

On the other hand, if $\left|a_{n}-s\right|>b_{n}$, and we know also that $b_{n} \geqslant M>M / 4>|s-t|$ due to (2.6),

$$
\begin{aligned}
\inf _{n}\left(1+\frac{(s-t)^{2}}{\left(a_{n}-s\right)^{2}+b_{n}^{2}}+\frac{2(s-t)\left(a_{n}-s\right)}{\left(a_{n}-s\right)^{2}+b_{n}^{2}}\right) & \geqslant \inf _{n}\left(1-\frac{2|s-t|\left|a_{n}-s\right|}{\left(a_{n}-s\right)^{2}+b_{n}^{2}}\right) \\
& \geqslant \inf _{n}\left(1-\frac{M\left|a_{n}-s\right|}{2\left(a_{n}-s\right)^{2}}\right) \\
& =\inf _{n}\left(1-\frac{M}{2\left|a_{n}-s\right|}\right) \geqslant 1-\frac{1}{2}=\frac{1}{2} .
\end{aligned}
$$

Therefore in any cases we have (3.4).

The two lemmas above are fundamental for the proof of the following proposition.

Proposition 3.6. Let $\iota: \mathcal{H}^{p}(E) \hookrightarrow \mathcal{H}^{q}(E)$ be continuous. If $\left\|\phi^{\prime}\right\|_{\infty}=\infty$, there exists a sequence $\left\{t_{n}\right\}_{n \in \mathbb{N}} \subset \mathbb{R}$ which goes to infinity such that

$$
\int_{\mathbb{R}}\left|\frac{\sin \left(\phi(x)-\phi\left(t_{n}\right)\right)}{x-t_{n}}\right|^{p} d x=\phi^{\prime}\left(t_{n}\right)^{p-1}
$$

Proof. Since $\left\|\phi^{\prime}\right\|_{\infty}=\infty$, there exist $\left\{t_{n}\right\}_{n \in \mathbb{N}}$ such that

$$
\lim _{n \rightarrow \infty} \phi^{\prime}\left(t_{n}\right)=\infty \text {. }
$$

Therefore

$$
\begin{aligned}
\int_{\mathbb{R}}\left|\frac{\sin \left(\phi(x)-\phi\left(t_{n}\right)\right)}{x-t_{n}}\right|^{p} d x & =\int_{\mathbb{R}}\left|\frac{\sin \left(\phi\left(x+t_{n}\right)-\phi\left(t_{n}\right)\right)}{x}\right|^{p} d x \\
& \geqslant \int_{-M / 4}^{M / 4}\left|\frac{\sin \left(\phi\left(x+t_{n}\right)-\phi\left(t_{n}\right)\right)}{x}\right|^{p} d x .
\end{aligned}
$$


If

$$
|x| \leqslant \frac{\pi}{24 \phi^{\prime}\left(t_{n}\right)}
$$

then

$$
\left|\phi\left(x+t_{n}\right)-\phi\left(t_{n}\right)\right| \leqslant \phi^{\prime}(s)|x| \leqslant 4 \phi^{\prime}\left(t_{n}\right)|x| \leqslant \frac{\pi}{6} \quad \text { where }\left|s-t_{n}\right| \leqslant|x| \leqslant M / 4 .
$$

Therefore, since

$$
|\sin (x)| \geqslant \frac{3}{\pi}|x| \text { for }|x| \leqslant \frac{\pi}{6}
$$

we have that

$$
\left|\sin \left(\phi\left(x+t_{n}\right)-\phi\left(t_{n}\right)\right)\right| \geqslant \frac{3}{\pi}\left|\phi\left(x+t_{n}\right)-\phi\left(t_{n}\right)\right| \text { when } x \text { satisfies (3.6) . }
$$

We can assume that $\frac{\pi}{24 \phi^{\prime}\left(t_{n}\right)}<M / 4$ if $t_{n}$ is large enough. Thus

$$
\begin{aligned}
\int_{\mathbb{R}}\left|\frac{\sin \left(\phi(x)-\phi\left(t_{n}\right)\right)}{x-t_{n}}\right|^{p} d x & \geqslant \int_{-\frac{\pi}{24 \phi^{\prime}\left(t_{n}\right)}}^{\frac{\pi}{24 \phi^{\prime}\left(t_{n}\right)}}\left|\frac{3}{\pi} \phi^{\prime}\left(t_{n}+s(x)\right)\right|^{p} d x \\
& \geqslant \int_{-\frac{\pi}{24 \phi^{\prime}\left(t_{n}\right)}}^{\frac{\pi}{24 \phi^{\prime}\left(t_{n}\right)}}\left|\frac{3}{2 \pi} \phi^{\prime}\left(t_{n}\right)\right|^{p} d x=\frac{1}{8}\left(\frac{3}{2 \pi}\right)^{p-1} \phi^{\prime}\left(t_{n}\right)^{p-1} .
\end{aligned}
$$

On the other hand

$$
\begin{aligned}
\int_{\mathbb{R}}\left|\frac{\sin \left(\phi(x)-\phi\left(t_{n}\right)\right)}{x-t_{n}}\right|^{p} d x & =\int_{\mathbb{R}}\left|\frac{\sin \left(\phi\left(x+t_{n}\right)-\phi\left(t_{n}\right)\right)}{x}\right|^{p} d x \\
& \leqslant \int_{-\frac{\pi}{24 \phi^{\prime}\left(t_{n}\right)}}^{\frac{24 \phi^{\prime}\left(t_{n}\right)}{x}}\left|\frac{\sin \left(\phi\left(x+t_{n}\right)-\phi\left(t_{n}\right)\right)}{x}\right|^{p}+2 \int_{\frac{\pi}{24 \phi^{\prime}\left(t_{n}\right)}}^{\infty} \frac{1}{x^{p}} \\
& \leqslant\left(4 \phi^{\prime}\left(t_{n}\right)\right)^{p} \frac{\pi}{12 \phi^{\prime}\left(t_{n}\right)}+\frac{(24)^{p-1} 2}{(p-1) \pi^{p-1}} \phi^{\prime}\left(t_{n}\right)^{p-1} \\
& \leqslant C \phi^{\prime}\left(t_{n}\right)^{p-1},
\end{aligned}
$$

since we assumed that $\frac{\pi}{24 \phi^{\prime}\left(t_{n}\right)}<M$ for $t_{n}$ large enough.

Proof Necessity 2.4. First of all we prove the necessary condition for $\iota: \mathcal{H}^{p}(E) \hookrightarrow \mathcal{H}^{\infty}(E)$ to be continuous. Assume towards a contradiction that $\left\|\phi^{\prime}\right\|_{\infty}=\infty$. Then, as proved in Proposition 3.6 there exists a sequence $\left\{t_{n}\right\}_{n \in \mathbb{N}}$ such that (3.5) holds. Thanks to the boundedness of the embedding operator, we know that

$$
\left\|k_{t_{n}}\right\|_{\mathcal{H} \infty} \leqslant C\left\|k_{t_{n}}\right\|_{\mathcal{H}^{p}}
$$

$$
=C\left(\int_{\mathbb{R}}\left|\frac{\sin \left(\phi(x)-\phi\left(t_{n}\right)\right)}{\left(x-t_{n}\right)}\right|^{p} d x\right)^{1 / p}\left|E\left(t_{n}\right)\right| \leqslant \tilde{C}\left(\phi^{\prime}\left(t_{n}\right)\right)^{1-1 / p}\left|E\left(t_{n}\right)\right| .
$$

Therefore, $\forall t_{n}$,

$$
\tilde{C} \phi^{\prime}\left(t_{n}\right)^{1-1 / p}\left|E\left(t_{n}\right)\right| \geqslant\left\|k_{t_{n}}\right\|_{\mathcal{H}^{\infty}} \geqslant\left|\frac{k_{t_{n}}\left(t_{n}\right)}{E\left(t_{n}\right)}\right|=\frac{\phi^{\prime}\left(t_{n}\right)}{\pi}\left|E\left(t_{n}\right)\right| .
$$

Consequently we would say that

$$
\left|\phi^{\prime}\left(t_{n}\right)\right| \leqslant C
$$

but it is impossible. For this reason $\left\|\phi^{\prime}\right\|_{\infty}<\infty$.

Let us move on to the general statement for $q<\infty$. As done before, let us assume that $\left\|\phi^{\prime}\right\|_{\infty}=\infty$. Then, as proved in Proposition 3.6 there exist $\left\{t_{n}\right\}_{n \in \mathbb{N}}$ such that (3.5) holds. We know that for every $t_{n},\left\|k_{t_{n}}\right\|_{\mathcal{H}^{q}} \leqslant$ $C\left\|k_{t_{n}}\right\|_{\mathcal{H}^{p}}$ and consequently,

$$
\begin{aligned}
C^{*} \phi^{\prime}\left(t_{n}\right)^{1-1 / q} \leqslant\left(\int_{\mathbb{R}}\left|\frac{\sin \left(\phi(x)-\phi\left(t_{n}\right)\right)}{x-t_{n}}\right|^{q} d x\right)^{1 / q} & \leqslant C\left(\int_{\mathbb{R}}\left|\frac{\sin \left(\phi(x)-\phi\left(t_{n}\right)\right)}{x-t_{n}}\right|^{p} d x\right)^{1 / p} \leqslant \tilde{C} \phi^{\prime}\left(t_{n}\right)^{1-1 / p}
\end{aligned}
$$


that is

$$
\phi^{\prime}\left(t_{n}\right)^{1 / p-1 / q} \leqslant \tilde{C^{*}} .
$$

However this last inequality cannot hold if $\phi^{\prime}\left(t_{n}\right)$ goes to infinity when $n$ goes to infinity. For this reason $\left\|\phi^{\prime}\left(t_{n}\right)\right\|<\infty$.

REMARK 3.7 The proof of the necessity condition is more complicated than the same proof of [10]. However, it can be generalized to the case $1 / 2<p \leqslant 1$ as we show in the next section.

\section{Proof of Theorem 2.5}

In this section we prove that $\phi^{\prime}(x) \in L^{\infty}(\mathbb{R})$ is a necessary condition also for the continuity of the embedding $\iota: \mathcal{H}^{1} \hookrightarrow \mathcal{H}^{q}$ when the function $E$ satisfies (2.5) and (2.6). The sufficiency of Theorem 2.5 is equal to the proof of the sufficiency of Theorem 2.4 and for this reason we omit the proof.

It is clear that (2.5) implies that for every $t, s \in \mathbb{R}$ such that $\phi(t)=\phi(s)+\pi$,

$$
t-s=\pi / \phi^{\prime}(\zeta) \leqslant \pi / \delta, \text { where } s \leqslant \zeta \leqslant t .
$$

In the proof of the following lemma, we use the relations (3.3) and (3.4).

LEMMA 4.1. Let us assume that the function E satisfy (2.5) and (2.6). Furthermore, let us consider

$$
s, t \in \mathbb{R} \text { so that } \phi(t)=\phi(s)+\pi \text {. }
$$

Then

$$
\int_{\mathbb{R}}\left|\frac{\sin (\phi(x)-\phi(s))(t-s)}{(x-t)(x-s)}\right| d x \leqslant C\left(\left|\log \left(\phi^{\prime}(t)\right)\right|+\mid \log \left(\phi^{\prime}(s) \mid+K\right) .\right.
$$

In particular, if $\left\{s_{n}\right\}_{n \in \mathbb{Z}} \subset \mathbb{R}$ and $\phi^{\prime}\left(s_{n}\right) \geqslant \phi^{\prime}\left(t_{n}\right) \rightarrow \infty$,

$$
\int_{\mathbb{R}}\left|\frac{\sin \left(\phi(x)-\phi\left(s_{n}\right)\right)\left(t-s_{n}\right)}{\left(x-t_{n}\right)\left(x-s_{n}\right)}\right| d x \leqslant C^{\prime} \log \left(\phi^{\prime}\left(s_{n}\right)\right) .
$$

Proof. We have to consider three possible situations. First of all, let us assume that

$$
\frac{\pi}{24} \frac{1}{\phi^{\prime}(s)}<\frac{(t-s)}{2}, \quad \frac{\pi}{24} \frac{1}{\phi^{\prime}(t)}<\frac{(t-s)}{2} .
$$

We split the integral in (4.1) in five parts:

$$
\begin{array}{r}
\int_{\mathbb{R}}\left|\frac{\sin (\phi(x)-\phi(s))(t-s)}{(x-t)(x-s)}\right| d x=\int_{\mathbb{R}}\left|\frac{\sin (\phi(s+\zeta)-\phi(s))(t-s)}{\zeta(t-s-\zeta)}\right| d \zeta \\
=\int_{-\infty}^{-\frac{\pi}{24} \frac{1}{\phi^{\prime}(s)}}+\int_{-\frac{\pi}{24} \frac{1}{\phi^{\prime}(s)}}^{\frac{\pi}{\phi^{\prime}(s)}}+\int_{\frac{\pi}{24} \frac{1}{\phi^{\prime}(s)}}^{t-s-\frac{\pi}{24} \frac{1}{\phi^{\prime}(t)}}+\int_{t-s-\frac{\pi}{24} \frac{1}{\phi^{\prime}(t)}}^{t-s+\frac{\pi}{24} \frac{1}{\phi^{\prime}(t)}}+\int_{t-s+\frac{\pi}{24} \frac{1}{\phi^{\prime}(t)}}^{\infty} \\
=(A)+(B)+(C)+(D)+(E) .
\end{array}
$$

We know that

$$
\begin{aligned}
(A) & =\int_{-\infty}^{-\frac{\pi}{24} \frac{1}{\phi^{\prime}(s)}}\left|\frac{\sin (\phi(s+\zeta)-\phi(s))(t-s)}{\zeta(t-s-\zeta)}\right| d \zeta \leqslant \int_{-\infty}^{-\frac{\pi}{24} \frac{1}{\phi^{\prime}(s)}}\left|\frac{1}{\zeta}+\frac{1}{t-s-\zeta} d \zeta\right| \\
& \leqslant\left|\log \left(\frac{\pi}{24} \frac{1}{\phi^{\prime}(s)}\right)\right|+|\log (C \pi / \delta)| .
\end{aligned}
$$


After a change of variables, we have the same estimate for $(E)$ :

$$
(E) \leqslant \log \left(C \phi^{\prime}(t)\right)+|\log (C \pi / \delta)|
$$

Let us move to $(B)$; we know that

$$
(B)=\int_{-\frac{\pi}{24} \frac{1}{\phi^{\prime}(s)}}^{\frac{\pi}{24} \frac{1}{\phi^{\prime}(s)}}\left|\frac{\sin (\phi(s+\zeta)-\phi(s))(t-s)}{\zeta(t-s-\zeta)}\right| d \zeta \leqslant \frac{\pi}{12} \frac{1}{\phi^{\prime}(s)} 4 \phi^{\prime}(s) 2=\pi \frac{2}{3} .
$$

The same calculations work also for $(D)$, for which we obtain the same estimate. Finally

$$
\begin{aligned}
(C) \leqslant & \int_{\frac{\pi}{24} \frac{1}{\phi^{\prime}(s)}}^{t-s-\frac{\pi}{24} \frac{1}{\phi^{\prime}(t)}}\left|\frac{1}{\zeta}+\frac{1}{t-s-\zeta}\right| d \zeta \\
\leqslant & \left|\log \left(\frac{\pi}{24} \frac{1}{\phi^{\prime}(s)}\right)\right|+\left|\log \left(t-s-\frac{\pi}{24} \frac{1}{\phi^{\prime}(s)}\right)\right| \\
& +\left|\log \left(\frac{\pi}{24} \frac{1}{\phi^{\prime}(t)}\right)\right|+\left|\log \left(t-s-\frac{\pi}{24} \frac{1}{\phi^{\prime}(t)}\right)\right| \\
\leqslant & 2 \log \left(C \phi^{\prime}(t)\right)+2 \log \left(C^{\prime} \phi^{\prime}(s)\right)+2|\log (\pi / \delta)| .
\end{aligned}
$$

Considering all the estimates, we proved relation (4.1).

Secondly, we assume that

$$
\frac{\pi}{24} \frac{1}{\phi^{\prime}(s)} \geqslant \frac{(t-s)}{2}, \quad \frac{\pi}{24} \frac{1}{\phi^{\prime}(t)} \geqslant \frac{(t-s)}{2} .
$$

We split the integral in (4.1) in four parts:

$$
\begin{aligned}
\int_{\mathbb{R}}\left|\frac{\sin (\phi(x)-\phi(s))(t-s)}{(x-s)(x-t)}\right| d x= & \int_{\mathbb{R}}\left|\frac{\sin (\phi(s+\zeta)-\phi(s))(t-s)}{\zeta(t-s-\zeta)}\right| d \zeta \\
& =\int_{-\infty}^{-\frac{(t-s)}{2}}+\int_{-\frac{(t-s)}{2}}^{\frac{(t-s)}{2}}+\int_{\frac{(t-s)}{2}}^{\frac{3(t-s)}{2}}+\int_{\frac{3(t-s)}{2}}^{\infty}=(A)+(B)+(C)+(D) .
\end{aligned}
$$

We know that

$$
\begin{aligned}
(A) & \leqslant \int_{-\infty}^{-\frac{(t-s)}{2}}\left|\frac{1}{\zeta}+\frac{1}{t-s-\zeta}\right| d \zeta \leqslant\left|\log \left(\frac{1}{2}(t-s)\right)\right|+\left|\log \left(\frac{3}{2}(t-s)\right)\right| \\
& \leqslant 2\left|\log \left(C \frac{\pi}{\phi^{\prime}(p)}\right)\right| \leqslant 2\left|\log \left(C \frac{3 \pi}{\phi^{\prime}(s)}\right)\right|
\end{aligned}
$$

After a change of variables, we have the same estimate for $(D)$ :

$$
(D) \leqslant 2 \log \left(C \phi^{\prime}(t)\right)
$$

Let us work on $(B)$; we know that

$$
(B)=\int_{-\frac{(t-s)}{2}}^{\frac{(t-s)}{2}}\left|\frac{\sin (\phi(s+\zeta)-\phi(s))(t-s)}{\zeta(t-s-\zeta)}\right| d \zeta \leqslant(t-s) \phi^{\prime}(p) 2 \leqslant \frac{\pi}{12} \frac{1}{\phi^{\prime}(s)} 4 \phi^{\prime}(s) 2=\frac{\pi 2}{3} .
$$

Analogously we estimate

$$
(C) \leqslant \frac{\pi}{12} \frac{1}{\phi^{\prime}(t)} 4 \phi^{\prime}(t) 2=\frac{\pi 2}{3} .
$$

Considering all the estimates, we proved relation (4.1).

Finally, we assume that

$$
\frac{\pi}{24} \frac{1}{\phi^{\prime}(s)}<\frac{(t-s)}{2}, \quad \frac{\pi}{24} \frac{1}{\phi^{\prime}(t)} \geqslant \frac{(t-s)}{2} .
$$


We split the integral in (4.1) in five parts:

$$
\begin{aligned}
\int_{\mathbb{R}}\left|\frac{\sin (\phi(x)-\phi(s))(t-s)}{(x-t)(x-s)}\right| d x=\int_{\mathbb{R}}\left|\frac{\sin (\phi(s+\zeta)-\phi(s))(t-s)}{\zeta(t-s-\zeta)}\right| d \zeta \\
=\int_{-\infty}^{-\frac{\pi}{24} \frac{1}{\phi^{\prime}(s)}}+\int_{-\frac{\pi}{24} \frac{1}{\phi^{\prime}(s)}}^{\frac{\pi}{24} \frac{1}{\phi^{\prime}(s)}}+\int_{\frac{\pi}{24} \frac{1}{\phi^{\prime}(s)}}^{(t-s) / 2}+\int_{(t-s) / 2}^{3(t-s) / 2}+\int_{3(t-s) / 2}^{\infty}=(A)+(B)+(C)+(D)+(E) .
\end{aligned}
$$

The only integral we have not already considered, is $(C)$ :

$$
\begin{aligned}
(C) & \leqslant \int_{\frac{\pi}{24} \frac{1}{\phi^{\prime}(s)}}^{(t-s) / 2}\left|\frac{1}{\zeta}+\frac{1}{t-s-\zeta}\right| d \zeta \\
& \leqslant 2 \log \left(\frac{t-s}{2}\right)+2\left|\log \left(\frac{\pi}{24} \frac{1}{\phi^{\prime}(s)}\right)\right|\left|\log \left(t-s-\frac{\pi}{24} \frac{1}{\phi^{\prime}(s)}\right)\right| \\
& \leqslant 2 \log (C \pi / \delta)+2\left|\log \left(\frac{\pi}{24} \frac{1}{\phi^{\prime}(s)}\right)\right| .
\end{aligned}
$$

Considering all the estimates, we proved relation (4.1) also in this final case.

Proof Necessity 2.5. Let us assume towards a contradiction that $\left\|\phi^{\prime}\right\|_{\infty}=\infty$. Then, there exists a sequence of points $\left\{s_{n}\right\}$ such that $\phi^{\prime}\left(s_{n}\right) \rightarrow \infty$ as $n$ goes to infinity and $E$ satisfies (2.5). First of all, we consider the function

$$
\Lambda_{n}(x):=k_{s_{n}}(x)+\frac{\left|E\left(s_{n}\right)\right|}{\left|E\left(t_{n}\right)\right|} k_{t_{n}}(x)=\frac{|E(x)|\left|E\left(s_{n}\right)\right|}{\pi} \frac{\sin \left(\phi(x)-\phi\left(s_{n}\right)\right)\left(t_{n}-s_{n}\right)}{\left(x-t_{n}\right)\left(x-s_{n}\right)},
$$

where $s_{n}, t_{n} \in \mathbb{R}$ so that $\phi\left(t_{n}\right)=\phi\left(s_{n}\right)+\pi$. Thanks to Lemma 4.1,

$$
\left\|\Lambda_{n}\right\|_{\mathcal{H}^{1}} \leqslant C\left|E\left(s_{n}\right)\right| \log \left(\phi^{\prime}\left(s_{n}\right)\right) \text {, when } \phi^{\prime}\left(s_{n}\right)>\phi^{\prime}\left(t_{n}\right) .
$$

If, on the other hand, $\phi^{\prime}\left(s_{n}\right) \leqslant \phi^{\prime}\left(t_{n}\right)$, we consider

$$
\tilde{\Lambda}_{n}(x):=k_{t_{n}}(x)+\frac{\left|E\left(t_{n}\right)\right|}{\left|E\left(s_{n}\right)\right|} k_{s_{n}}(x)=\frac{|E(x)|\left|E\left(t_{n}\right)\right|}{\pi} \frac{\sin \left(\phi(x)-\phi\left(t_{n}\right)\right)\left(t_{n}-s_{n}\right)}{\left(x-t_{n}\right)\left(x-s_{n}\right)},
$$

for which

$$
\left\|\tilde{\Lambda}_{n}\right\|_{\mathcal{H}^{1}} \leqslant C\left|E\left(t_{n}\right)\right| \log \left(\phi^{\prime}\left(t_{n}\right)\right) \text { holds. }
$$

For sake of clarity, from now on, we assume $\phi^{\prime}\left(s_{n}\right) \geqslant \phi^{\prime}\left(t_{n}\right)$.

We start by the proof of the continuity of $\mathcal{H}^{1}(E) \hookrightarrow \mathcal{H}^{\infty}(E)$. Thanks to the boundedness of the embedding operator, we know that

$$
\left\|\Lambda_{n}\right\|_{\mathcal{H}^{\infty}} \leqslant C\left\|\Lambda_{n}\right\|_{\mathcal{H}^{1}}=C^{\prime}\left|E\left(s_{n}\right)\right| \log \left(\phi^{\prime}\left(s_{n}\right)\right) .
$$

Therefore, $\forall s_{n}$,

$$
C^{\prime}\left|E\left(s_{n}\right)\right| \log \left(\phi^{\prime}\left(s_{n}\right)\right) \geqslant\left\|\Lambda_{n}\right\|_{\mathcal{H}^{\infty}} \geqslant\left|\frac{\Lambda_{n}\left(s_{n}\right)}{E\left(s_{n}\right)}\right|=\frac{\phi^{\prime}\left(s_{n}\right)}{\pi}\left|E\left(s_{n}\right)\right|,
$$

which is impossible. Consequently,

$$
\left|\phi^{\prime}\left(s_{n}\right)\right| \leqslant C \text {, and }\left\|\phi^{\prime}\right\|_{\infty}<\infty .
$$

Let us move on to the general statement for $q<\infty$. We know that for every $n,\left\|\Lambda_{n}\right\|_{\mathcal{H}^{q}} \leqslant C\left\|\Lambda_{n}\right\|_{\mathcal{H}^{1}}$. We know also that, when $n$ is large enough,

$$
\begin{gathered}
\left\|\Lambda_{n}\right\|_{\mathcal{H}^{q}}^{q}=\left|E\left(s_{n}\right)\right|^{q} \int_{\mathbb{R}}\left|\frac{\sin \left(\phi(x)-\phi\left(s_{n}\right)\right)\left(s_{n}-t_{n}\right)}{\left(x-s_{n}\right)\left(x-t_{n}\right)}\right|^{q} d x \\
\geqslant \int_{-\frac{\pi}{24 \phi^{\prime}\left(s_{n}\right)}}^{\frac{\pi}{24 \phi^{\prime}\left(s_{n}\right)}}\left|\frac{3}{\pi} \phi^{\prime}\left(s_{n}+\zeta(x)\right)\right|^{q} d x\left|\frac{t_{n}-s_{n}}{t_{n}-s_{n}+\frac{\pi}{24 \phi^{\prime}\left(s_{n}\right)}}\right|^{q} \\
\quad \geqslant \int_{-\frac{\pi}{24 \phi^{\prime}\left(s_{n}\right)}}^{\frac{\pi}{24 \phi^{\prime}\left(s_{n}\right)}}\left|\frac{3}{4 \pi} \phi^{\prime}\left(s_{n}\right)\right|^{q} d x=\frac{1}{8}\left(\frac{3}{4 \pi}\right)^{q-1} \phi^{\prime}\left(s_{n}\right)^{q-1} .
\end{gathered}
$$


Therefore

$$
C^{\prime}\left|E\left(s_{n}\right)\right| \phi^{\prime}\left(s_{n}\right)^{1-1 / q} \leqslant\left|E\left(s_{n}\right)\right| \int_{\mathbb{R}}\left|\frac{\sin \left(\phi(x)-\phi\left(s_{n}\right)\right)\left(s_{n}-t_{n}\right)}{\left(x-s_{n}\right)\left(x-t_{n}\right)}\right| d x \leqslant \quad C^{\prime \prime}\left|E\left(s_{n}\right)\right| \log \left(\phi^{\prime}\left(s_{n}\right)\right) .
$$

However this last inequality cannot hold if $\phi^{\prime}\left(s_{n}\right)$ goes to infinity when $n$ goes to infinity. Consequently $\left\|\phi^{\prime}\right\|_{\infty}<\infty$.

REMARK 4.2 We note that the previous proof with some natural changes shows also that Theorem 2.5 holds even when $1 / 2<p<1$.

\section{Conclusion}

In our opinion, the most innovative part of these notes is the fourth section where we proved that the boundedness of the derivative of the phase function is a necessary condition for the boundedness of the embedding operator also when $p=1$, if conditions (2.5) and (2.6) hold. We strongly believe that this result can be further improved, considering even $p \leqslant 1 / 2$. In order to do this, we would need an atomic description of the $p$-de Branges spaces, probably using Clark's measures.

Conflict of interest: The author states no conflict of interest. No data-set was used.

\section{References}

[1] N.B.Andersen, Entire $L^{p}$-functions of exponential type, Expo. Math., Elsevier, Munich, 32 (2014), 3, 199-220.

[2] A.D. Baranov, Differentiation in the Branges spaces and embedding theorems, J. Math. Sci. (N.Y.), Springer, New York, 101 (2000), 2881-2913.

[3] A.D. Baranov, Estimate of the $L^{p}$-norms of derivatives in spaces of entire functions, J. Math. Sci. (N.Y.), Springer, New York, 129 (2005), 3927-3943.

[4] A.D. Baranov, Bernstein-type inequalities for shift-coinvariant subspaces and their applications to Carleson embeddings, J. Funct. Anal., Elsevier B. V., Amsterdam, 223 (2005), 1, 116-146.

[5] J.A. Cima, W.T. Ross, The backward shift on the Hardy space, Mathematical Surveys and Monographs, American Mathematical Society, 792000.

[6] L. de Branges, Hilbert spaces of entire functions, Prentice-Hall, 1968.

[7] P.L. Duren, Theory of $H^{p}$ spaces, Pure and Applied Mathematics, Elsevier, 381970.

[8] K.M. Dyakonov, Entire functions of exponential type and model subspaces in $H^{p}$, J. Math. Sci. (N.Y.), Springer, New York, 71 (1994), 2222-2233.

[9] K.M. Dyakonov, Moduli and arguments of analytic functions from subspaces of $H^{p}$ that are invariant for the backward shift operator, Sib. Math. J., Pleiades Publishing, Ltd., Road Town, 31 (1990), 926-939.

[10] K.M. Dyakonov, Continuous and compact embeddings between star-invariant subspaces, Complex Anal. Oper. Theory, Birkhäuser, Basel, 113 (2000), 65-76.

[11] S. Garcia, J. Mashreghi, W.T. Ross, Introduction to model spaces and their operators, Cambridge Studies in Advanced Mathematics, Cambridge University Press, Cambridge, 2016.

[12] F.F. Gonçalves, Interpolation formulas with derivatives in de Branges spaces, Trans. Amer. Math. Soc. 369 (2017) $2,805-832$.

[13] F.F. Gonçalves, F. Littmann, Mean convergence of entire interpolations in weighted space, Complex Anal. Oper. Theory, Birkhäuser, Basel, 15 (2021) 5, Paper No. 90.

[14] B.Y. Levin, Lectures on entire functions, Translations of Mathematical Monographs, American Mathematical Society, 150 1996.

[15] B.I.A. Levin, Distribution of Zeros of Entire Functions, Translations of mathematical monographs, American Mathematical Society, 1980.

[16] N. K. Nikolski, Operators, functions and systems: An easy reading. Volume I: Hardy, Hankel, and Toeplitz, Mathematical Surveys and Monographs, American Mathematical Society, 922002.

[17] R. Romanov, Canonical systems and de Branges spaces, arXiv: Complex variables, 2014. 